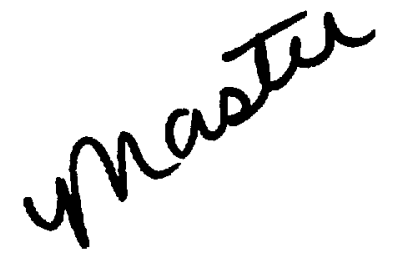

LA-8473-MS

Informal Report

UC-11

Issued: August 1980

\title{
Compilation of Elemental Concentration Data for the United States Geological Survey's Six Geochemical Exploration Reference Materials
}

\author{
Ernest S. Gladney
}

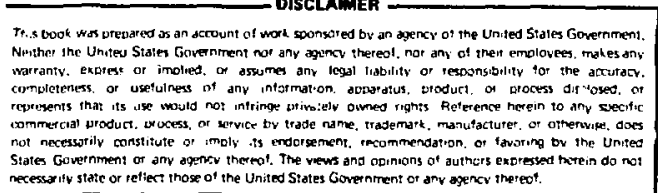


COMPILATION OF ELEMENTAL CONCENTRATION DATA FOR THE UNITED STATES GEOLOGICAL SURVEY'S SIX GEOCHEMICAL

EXPLORATION REFERENCE MATERIALS

by

Ernest S. Gladney

\begin{abstract}
Elemental composition data on USGS GXR1 - GXR6 have been collected and summarized from 13 journal papers and reports. Data from one report concerning the influence of rock dissolution method on subsequent atomic absorption analysis are summarized for aluminum, silver, calcium, iron, and manganese.
\end{abstract}

\title{
INTRODUCTION
}

This document is intended to provide an updated and more complete survey of literature values reported for the United States Geological Survey's (USGS) Geochemical Exploration Reference Materials (GXR). These six silicate standard materials are beirig made available through the Denver, Co, office of the USGS. An extensive open file report of original data has been made available (1), but none of these data were summarized until the publication of our Laboratory's original measurement of elemental concentrations in the GXR materials (2). Allcott and Lakin (3) of the USGS have also published their emission spectrographic and atomic absorption data for the GXR materials. This report will summarize data from references 1 and 3 which was not included in our earlier publication (2).

The Environmental Surveillance Group uses standard reference materials from the National Bureau of Standards, the Environmental Protection Agency, 
and the Canadian Centre for Mineral and Energy Technology, in addition to those from the USGS as an integral part of our quality assurance program for elemental measurements. High quality "certified" and "recommended" values are available for a number of constituents in these matrices. However, many elements are not reported by the issuing agencies. If enough reliable data from other investigators can be accumuiated and summarized, we can use these mean values to extend the range of our quality assurance program within the standard materials already available.

\section{DATA COMPILATION}

The GXR rock designations, names, descriptions and sources are shown in Table I. In addition to the USGS data $(1,3)$, eighteen major journals in analytical chemistry and geochemistry shown in Table il have been exhaustively surveyed in an effort to find original analytical data on these six USGS standards. Only ten articles containing original data, in addition to the three sources mentioned above, were located. Since the available data are so limited, no statistical treatment of them are possible.

A summary of the recommended vaiues developed at this Laboratory and published in Ref. 2 is presented in Table III. These elemental concentrations were determined by a combination of analytical procedures including instrumental and radiochemical neutron activation, flame and flameless atomic absorption, thermal neutron capture gamma-ray spectrometry, and delayed neutron assay. A summary of the available data from Allcott and Lakin $(1,3)$ for these elements was presented in the original paper and will not be reproduced here. Data from Allcott and Lakin $(1,3)$ for elements not measured in our previous study are presented in Table IV along with the compiled data from ten other investigations. All data in Tables III and IV must be used 
with caution since there is only limited basis for judgment of their quality.

The data from 109 laboratories tabulated by Allcott and Lakin (1) are given by both analytical technique used and dissolution method employed (where appropriate). Our previous paper presented a comparison among data taken by various analytical methods (2). In Tables $V$ to $X$ is my summary of the influence of dissolution method on atomic absorption determination of $A 1$, $\mathrm{Ag}, \mathrm{Ca}, \mathrm{Fe}$, and $\mathrm{Mn}$ in the GXR materials. Table $\mathrm{V}$ contains the dissolution method code while Tables VI to $X$ are means \pm one standard deviation of the raw data given by $A l l c o t t$ and Lakin ( 1 ). For $A l$, methods $M$ and $R$ yield consistently low results for all six samples. The use of $\mathrm{HClO}_{4}$ in sample preparation for Al determination is apparently the problem, since HF is successfully used in method $\mathrm{L}$. For $\mathrm{Ag}$, only method $\mathrm{B}$ yields low results and then only for the higher concentration samples. Insufficient contact time may be the explanation. For $\mathrm{Ca}$, only method $\mathrm{I}$ is completely inadequate, while several methods indicate difficulty with the very high or relatively low Ca levels encountered in GXR-3 and GXR-6, respectively. For Fe, methods $A$ and $E$ yield low results for the highest concentration samples (GXRI and 3) but all methods seem equivalent for Fe concentrations of less than $6 \%$. Several methods did not work well for the determination of Mn at percent levels encountered in GXR-3. Methods $A, E, I, M$, and 0 yielded low values, while method $P$ had high results. With the exception of method $A$ for GXR-6, all methods gave roughly equivalent data for the other GXR samples.

\section{Acknowledgements}

I thank Mary Lou Keigher for her assistance with the preparation of this manuscript. 


\section{REFERENCES}

1. G. H. Allcott, H. W. Lakin, "Tabulation of Geochemical Data Furnished by 109 Laboratories for Six Geochemical Exploration Reference Samples," USGS, Open File Report 78-163, 199 pp., 1978.

2. E. S. Gladney, D. R. Perrin, J. W. Owens, D. Knab, "Elemental Concentrations in the United States Geological Survey's Geochemical Exploration Reference Samples - A Review," Anal. Chom., 51: 1557-1569 (1979).

3. G. H. All cott, H. W. Lakin, "The Homogeneity of Six Geochemical Exploration Reference Samples," Geochemical Exploration 1974, Elseiver, New York, 1975, pp. 659-681.

4. J. G. Viets, "Determination of Silver, Bismuth, Cadmium, Copper, Lead, and Zinc in Geological Materials by Atomic Absorption Spectrometry with Tricaprylylmethylammonium Chloride," Anal. Chem., 50: 1097-1101 (1978).

5. J. M. Motooka, E. L. Mosier, S. J. Sutley, J. G. Viets, "Inductioncoupled Plasma Determination of $\mathrm{Ag}$, $\mathrm{Au}, \mathrm{Bi}, \mathrm{Cd}, \mathrm{Cu}, \mathrm{Pb}$, and $\mathrm{Zn}$ in Geological Materials Using a Selective Extraction Technique - Preliminary Investigation," Applied Spectroscopy, 33, 456-460 (1979).

6. S. Terashima, "Determination of Arsenic in Rocks, Sediments, and Minerals by Arsine Generation and Atomic Absorption Spectrometry," Anal. Chim. Acta, 86: 43-51 (1976).

7. S. Terashima, "Rapid Determination of Total Carbon and Sulfur in Geological Materials by Combustion and Infrared Absorption Photometry," Anal. Chim. Acta, 101: 25-31 (1978).

8. R. F. Sanzolone, T. T. Chao, G. L. Crenshaw, "Atomic Absorption Spectrometric Determination of Cobalt, Nickel, and Copper in Geological Materials with Matrix Masking and Chelation-Extraction," Anal. Chim. Acta, 105: 247-253 (1979).

9. R. F. Sanzolone, T. T. Chao, "Atomic Absorption Spectrometric Determination of Copper, Zinc, and Lead in Geological Materials," Anal. Chim. Acta, 86: 163-168 (1976).

10. E. P. Welsch, T. T. Chao, "Determination of Trace Amounts of Antimony in Geological Materials by Atomic Absorption Spectrometry," Anal. Chim. Acta, 76: 65-69 (1975).

11. D. Knab, E. S. Gladney, "Determination of Selenium in Environmental Materials by Neutron Activation and Inorganic Ion Exchange," Anal. Chem., 52: 825-828 (1980).

12. E. P. Welsch, T. T. Chao, "Determination of Trace Amounts of Tin in Geological Materials by Atomic Absorption Spectrometry," Anal. Chim. Acta, 82: 337-342 (1976). 


\section{REFERENCES (Continued)}

13. T. T. Chao, R. F. Sanzolone, A. E. Hubert, "Flame and Flameless Atomic Absorption Determination of Tellerium in Geological Materials," Anal. Chim. Acta 96: 251-257 (1978). 
TABLE I: SIX USGS GXR MATERIALS

Standard Designation

GXR-1

GXR-2

GXR-3

GXR-4

GXR-5

GXR-6
DESCRIPTION: SOURCE

Jasperoid: Drum Mountains, Utah

Soil: Park Cit', Utah

Fe-Mn-W-rich Hot Spring Deposit:

Humboldt County, Nevada

Porphyry Copper Mill Heads: Utah

Soil (B zone): Somerset County, Maine

Soil (B zone): Davidson County, North Carolina 
TABLE II: ARCHIVAL JOURNALS SURVEYED

Journal

Analyst

Analytical Chemistry

Analytical Chimica Acta

Analytical Letters

Applied Spectroscopy

Atomic Absorption Newsletter

Chemical Geology

Environmental Science \& Technology

Geochimica et Cosmochimica Acta

Geostandards Newsletter

International Journal of Applied

Radiation and Isotopes

Journal of the Association of Official

Analytical Chemists

Journal of Radioanalytical Chemistry

Microchemical Journal

Nuciear Instruments and Methods

Radiochemical and Radioanalytical

Letters

Talanta

X-Ray Spectrometry
Volume Numbers Examined

97-104

43-51

53-106

$5-12$

32-33

$11-18$

$13-25$

$6-13$

$31-43$

1-3

23-30

$55-62$

$11-49$

$17-24$

114-155

9-39

$19-26$

7 
TABLE III

ELEMENTAL CONCENTRATIONS IN USGS GEOCHEMICAL EXPLORATION REFERENCE SAMPLES DETERMINE BY LASL (2)

(ppm unless $\%$ indicated)

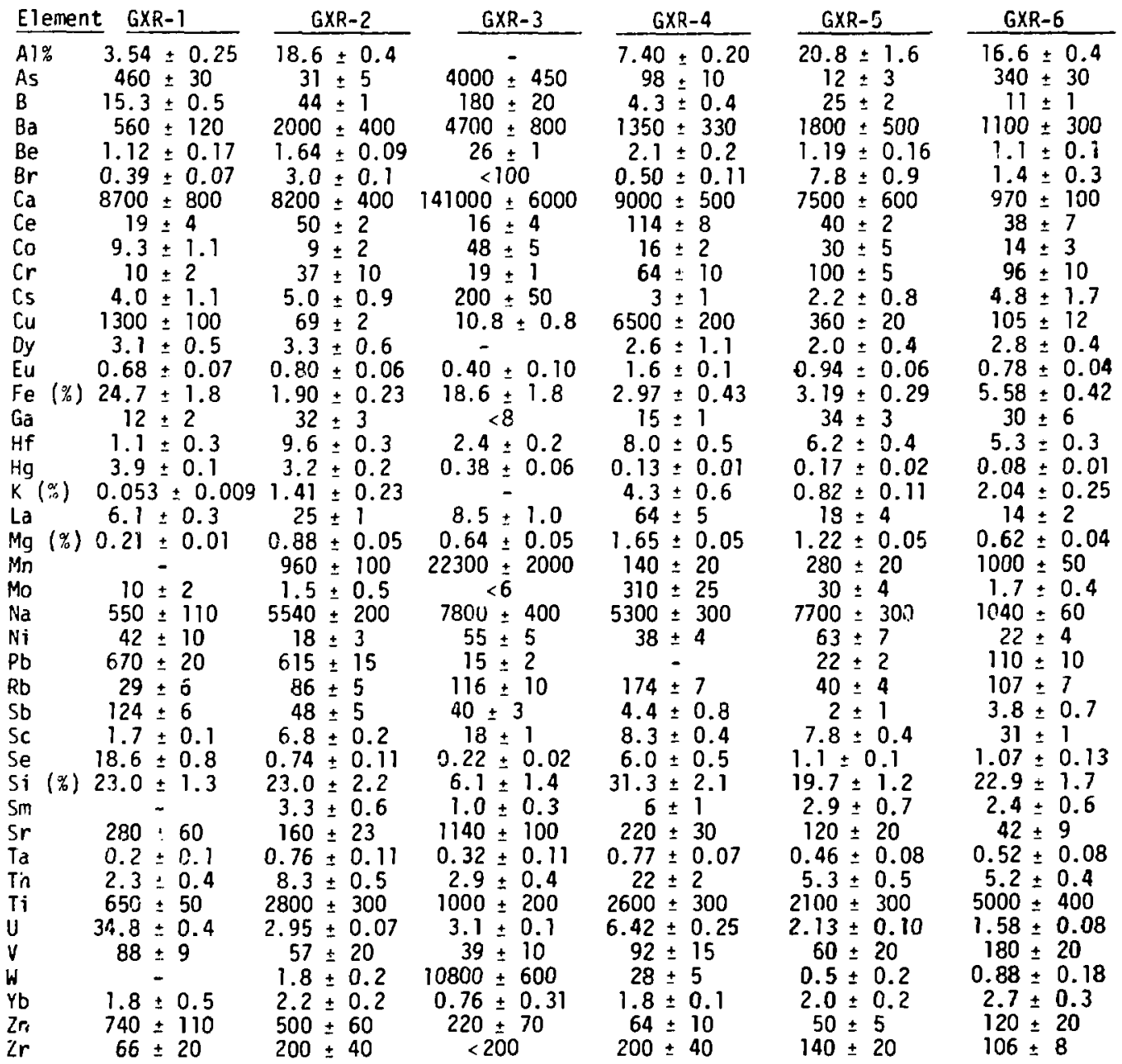


TABLE IV: ELEMENTAL CONCENTRATIONS IN USGS GXR SAMPLES

DETERMINED BY VARIOUS INVESTIGATORS

(ppm unless \% indicated)

\begin{tabular}{|c|c|c|c|c|c|c|c|c|}
\hline Ele. & Ref. & $\begin{array}{l}\text { Anal. } \\
\text { Tech. } \underline{\mathrm{a}}\end{array}$ & GXR-1 & GXR-2 & GXR-3 & GXR-4 & GXR-5 & GXR-6 \\
\hline \multirow[t]{6}{*}{$\mathrm{Ag}$} & 1 & A & 29 & 16 & 2.8 & 4.3 & 1.9 & 1.9 \\
\hline & & B & 28 & 15 & $<3$ & $<3$ & $<2$ & $<2$ \\
\hline & 3 & c & $29 \pm 9$ & $15 \pm 2$ & $<0.5$ & $3.6 \pm 1.1$ & $<0.5$ & $<0.5$ \\
\hline & & $D$ & $29 \pm 1$ & $13 \pm 1$ & $1.7 \pm 0.2$ & $3.6 \pm 0.2$ & $1.3 \pm 0.1$ & $1.1 \pm 0.1$ \\
\hline & 4 & A & $32.6 \pm 0.9$ & $17.4 \pm 0.6$ & $0.06 \pm 0.01$ & $3.64 \pm 0.05$ & $0.82 \pm 0.03$ & $0.29 \pm 0.02$ \\
\hline & 5 & c & $32.6 \pm 1.8$ & $17.7 \pm 0.5$ & $0.17 \pm 0.04$ & $3.8 \pm 0.1$ & $0.77 \pm 0.02$ & $0.35 \pm 0.03$ \\
\hline As & 6 & A & 436 & 23.3 & 3980 & 103 & 11.8 & 297 \\
\hline \multirow[t]{6}{*}{$\mathrm{Au}$} & 1 & A & $3.4 \pm 0.9$ & $0.10 \pm 0.09$ & $0.12 \pm 0.16$ & $0.65 \pm 0.28$ & $0.08 \pm 0.10$ & $0.13 \pm 0.07$ \\
\hline & & $E$ & $3.9 \pm 0.6$ & $<0.3$ & $<0.3$ & 0.75 & $<0.3$ & $<0.3$ \\
\hline & & $\mathrm{F}$ & 3.2 & $0.03 \rightarrow 0.55$ & $0.03 \rightarrow 0.16$ & 0.6 & $0.01 \rightarrow 0.43$ & $0.11 \rightarrow 0.41$ \\
\hline & 3 & c & $<10$ & $<10$ & $<10$ & $<10$ & $<10$ & $<0$ \\
\hline & & $D$ & $3.1 \pm 0.2$ & $0.04 \pm 0.01$ & $0.06 \pm 0.01$ & $0.64 \pm 0.06$ & $0.13 \pm 0.03$ & $0.08 \pm 0.03$ \\
\hline & 5 & c & $3.4 \pm 0.2$ & - & $0.6 \pm 0.1$ & $0.65 \pm 0.19$ & - & $0.12 \pm 0.01$ \\
\hline \multirow[t]{4}{*}{$\mathrm{Bi}$} & 1 & $F$ & 1000 & $<20$ & 105 & 50 & 70 & 60 \\
\hline & 3 & C & $540 \pm 97$ & $<10$ & $<10$ & $14 \pm 2$ & $<10$ & $=10$ \\
\hline & 4 & $A$ & $1725 \pm 24$ & 0.4 & $<0.2$ & $21.2 \pm 0.8$ & 0.4 & 0.2 \\
\hline & 5 & $c$ & $1204 \pm 23$ & - & $23 \pm 4$ & $18.8 \pm 0.4$ & - & - \\
\hline \multirow[t]{2}{*}{$c(\%)$} & 1 & G & $0.11 \rightarrow 0.53$ & $2.5 \rightarrow 5.5$ & $1.2 \pm 0.2$ & $<0.05 \rightarrow 3.8$ & $1.7 \pm 0.2$ & $0.15 \pm 0.02$ \\
\hline & 7 & G & 0.17 & 2.64 & 1.33 & 0.06 & 1.65 & 0.18 \\
\hline
\end{tabular}




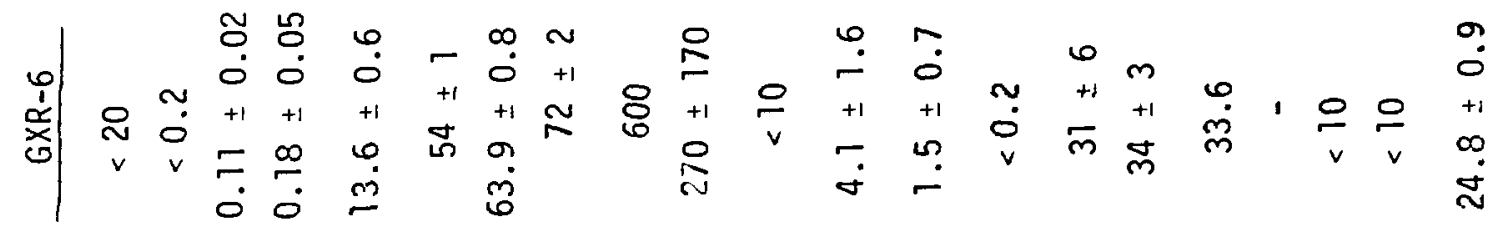

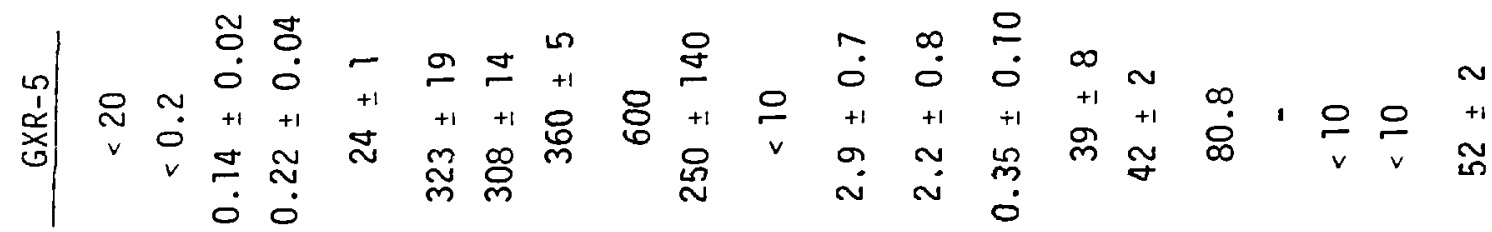

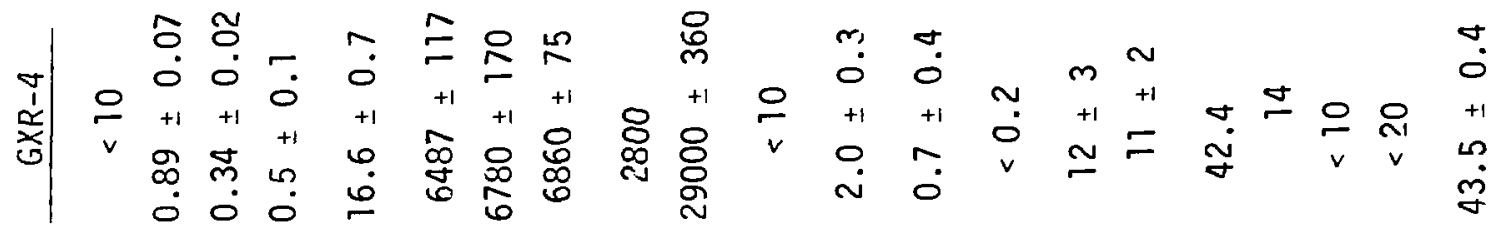

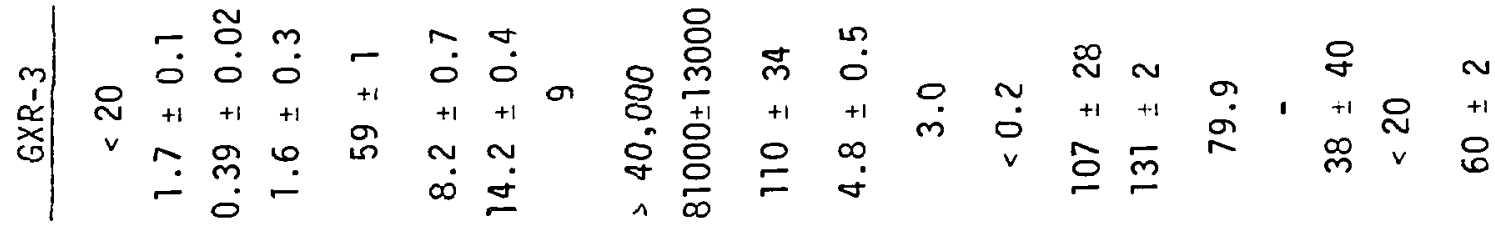

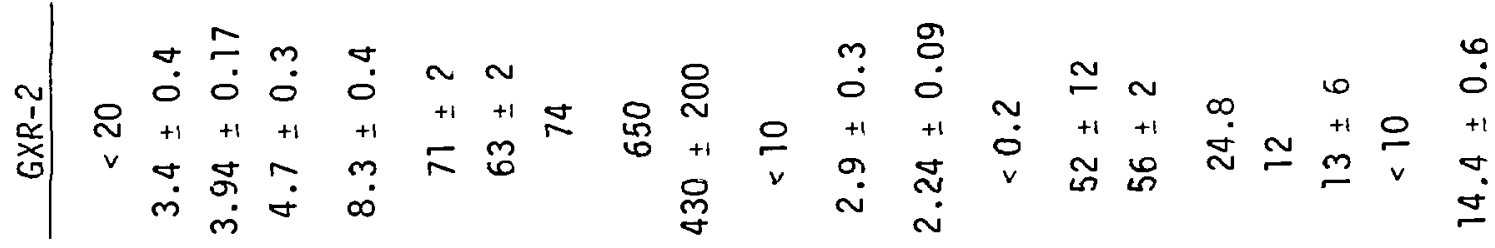

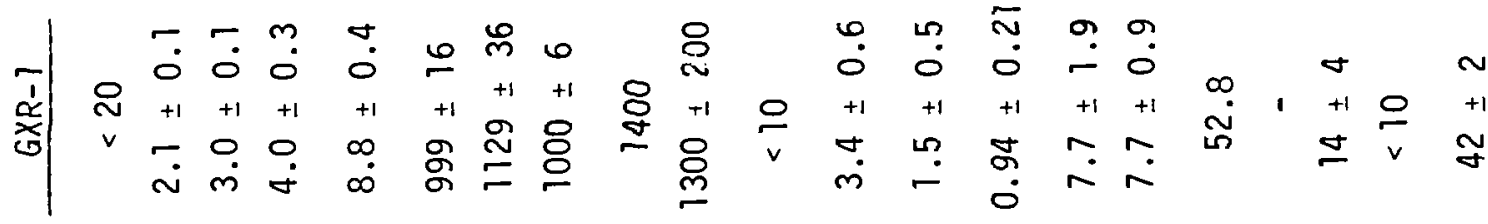

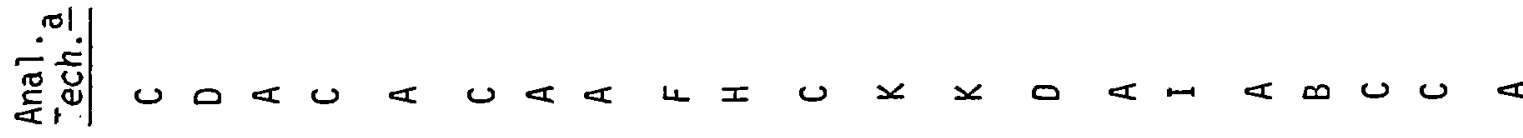

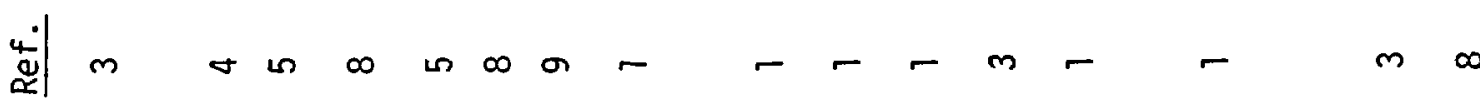

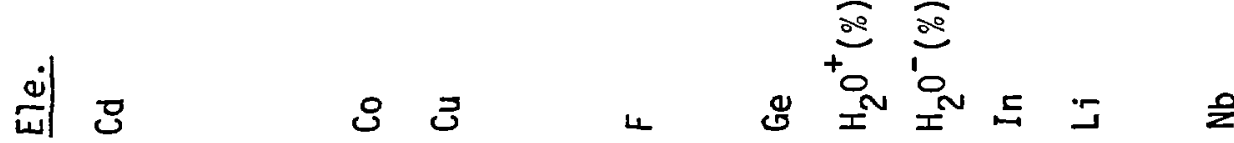




\begin{tabular}{|c|c|c|c|c|c|c|c|c|}
\hline Ele. & Ref. & $\begin{array}{l}\text { Anal. } \\
\text { Tech. } \\
\end{array}$ & GXR-1 & GXR-2 & GXR-3 & GXR-4 & GXR-5 & GXR-6 \\
\hline \multirow[t]{3}{*}{ P } & 1 & B & $440 \pm 220$ & $440 \pm 350$ & $<40 \rightarrow 2000$ & 480 & $300 \pm 100$ & $300 \pm 200$ \\
\hline & & $\mathrm{F}$ & $400 \pm 200$ & $300 \pm 100$ & $700 \pm 50$ & $900 \pm 100$ & $130 \pm 90$ & $220 \pm 170$ \\
\hline & & K & 700 & 1100 & 1000 & 1000 & 740 & 1000 \\
\hline \multirow[t]{2}{*}{$\mathrm{Pb}$} & 5 & C & $692 \pm 32$ & $651 \pm 38$ & $23 \pm 2$ & $41 \pm 1$ & $15 \pm 2$ & $96 \pm 7$ \\
\hline & 9 & A & $825 \pm 17$ & $725 \pm 8$ & $20 \pm 1$ & $54 \pm 1$ & $16 \pm 1$ & $100 \pm 2$ \\
\hline \multirow[t]{2}{*}{$\mathrm{Pd}$} & 1 & c & $<4$ & $<4$ & $<4$ & $<4$ & $<4$ & $<4$ \\
\hline & & $F$ & - & - & 1.5 & - & - & - \\
\hline Pt & 1 & $F$ & 0.3 & 0.1 & - & 0.1 & 0.05 & 0.1 \\
\hline \multirow[t]{3}{*}{$S$} & 1 & B & $4100 \pm 800$ & - & $5000 \pm 600$ & $21900 \pm 4400$ & - & - \\
\hline & & $\mathrm{J}$ & $2600 \pm 200$ & - & $2300 \pm 400$ & $17500 \pm 800$ & - & - \\
\hline & 7 & G & 2436 & 315 & 2420 & 17600 & 278 & 131 \\
\hline Sb & 10 & A & $98 \pm 5$ & $44 \pm 2$ & $21.9 \pm 0.8$ & $5.1 \pm 0.3$ & $1.6 \pm 0.1$ & $2.8 \pm 0.3$ \\
\hline Se & 11 & $L$ & $19 \pm 1$ & $0.8 \pm 0.2$ & $0.22 \pm 0.03$ & $6.0 \pm 0.6$ & $1.2 \pm 0.2$ & $1.2 \pm 0.2$ \\
\hline \multirow[t]{6}{*}{ Sn } & 1 & A & 64 & $<1$ & $<1$ & 6 & 1 & 2 \\
\hline & & B & $34 \rightarrow 470$ & $1 \rightarrow 120$ & $<1 \rightarrow<50$ & $2 \rightarrow 160$ & $<1 \rightarrow 350$ & $3-1080$ \\
\hline & & C & $64 \pm 30$ & $<10$ & $<10$ & $<10$ & $<10$ & $<10$ \\
\hline & & $F$ & $60 \pm 32$ & $15 \pm 6$ & $108 \pm 68$ & $15 \pm 8$ & $12 \pm 7$ & $<0.15 \rightarrow 30$ \\
\hline & 3 & C & $63 \pm 11$ & $<10$ & $<10$ & $<10$ & $<10$ & $<10$ \\
\hline & 12 & A & $55 \pm 1$ & - & - & - & - & - \\
\hline \multirow[t]{3}{*}{ Te } & 1 & A & 16.1 & 3.3 & 0.11 & 1.2 & 0.15 & 0.08 \\
\hline & 3 & D & - & $<0.5$ & $<0.5$ & $1.0 \pm 0.4$ & $<0.5$ & $<0.5$ \\
\hline & 13 & $A$ & $8.7 \pm 0.1$ & $0.88 \pm 0.01$ & $0.009 \pm 0.002$ & $1.0 \pm 0.1$ & - & $0.016 \pm 0.001$ \\
\hline
\end{tabular}


Anal.

\begin{tabular}{|c|c|c|c|c|c|c|c|c|}
\hline Ele. & Ref. & Tech. $^{\mathrm{a}}$ & GXR-1 & $G \times R-2$ & GXR-3 & $G \times R-4$ & GXR-5 & GXR-6 \\
\hline T1 & 3 & 0 & $<0.02$ & $1.3 \pm 0.1$ & $4.2 \pm 0.4$ & $3.8 \pm 0.3$ & $0.4 \pm 0.1$ & $2.8 \pm 0.2$ \\
\hline \multirow[t]{2}{*}{ Y } & 1 & $c$ & $40 \pm 14$ & $15 \pm 4$ & $1 \varepsilon \pm 6$ & $14 \pm 5$ & $15 \pm 5$ & $15 \pm 6$ \\
\hline & 3 & $C$ & $57 \pm 11$ & $16 \pm 1$ & $16 \pm 2$ & $12 \pm 3$ & $16 \pm 3$ & $14 \pm 3$ \\
\hline \multirow[t]{2}{*}{$Z n$} & 5 & $C$ & $670 \pm 40$ & $425 \pm 18$ & $83 \pm 7$ & $63 \pm 3$ & $37 \pm 3$ & $104 \pm 12$ \\
\hline & 9 & $A$ & $675 \pm 8$ & $470 \therefore 12$ & $204 \pm 2$ & $76 \pm 2$ & $5 i \pm 2$ & $100 \pm 2$ \\
\hline
\end{tabular}

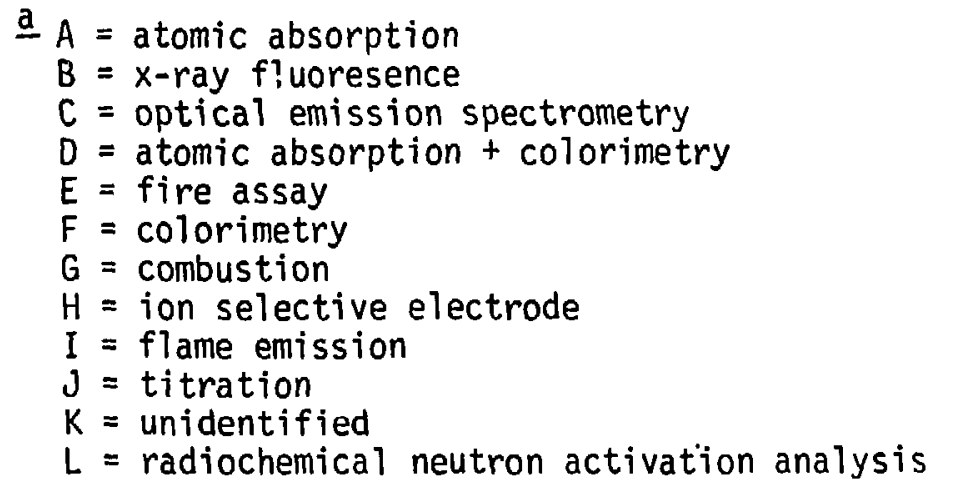


TABLE V: DISSOLUTION METHODS

\section{Letter Key}

A

B

C

D

E

$F$

G

H

I

$\mathrm{J}$

K

$\mathrm{L}$

M

N

0

$\mathrm{P}$

Q

$R$

$S$

T
Reagents Used for Sample Dissolution

Conc. $\mathrm{HNO}_{3}$

Dil. $\mathrm{HNO}_{3}$

$6 \mathrm{~N} \mathrm{HCl}$

$\mathrm{HF}$

$\mathrm{HClO}_{4}$

$\mathrm{Na}_{2} \mathrm{O}_{2}$ fusion

$\mathrm{LiBO}_{2}$ fusion

$\mathrm{HNO}_{3}+\mathrm{HCl}$

$\mathrm{HNO}_{3}+\mathrm{HClO}_{4}$

$\mathrm{HNO}_{3}+\mathrm{HF}$

$\mathrm{HNO}_{3}+\mathrm{HgNO}_{3}$

$\mathrm{HF}+\mathrm{HCl}$

$\mathrm{HF}+\mathrm{HClO}_{4}$

$\mathrm{HF}+\mathrm{HNO}_{3}+\mathrm{HCl}$

$\mathrm{HF}+\mathrm{HiNO}_{3}+\mathrm{H}_{2} \mathrm{SO}_{4}$

$\mathrm{HCl}+\mathrm{HNO}_{3}+\mathrm{KClO}_{4}$

$\mathrm{HCl}+\mathrm{HNO}_{3}+\mathrm{HClO}_{4}$

$\mathrm{HF}+\mathrm{HNO}_{3}+\mathrm{HClO}_{4}+\mathrm{HCl}$

$\mathrm{HF}+\mathrm{HClO}_{4}+\mathrm{HCl}$

$\mathrm{HF}+\mathrm{HNO}_{3}+\mathrm{HClO}_{4}$ 
TABLE VI: INFLUENCE OF DISSOLUTION METHOD ON THE DETERMINATION OF ALUMINUM IN GEOLOGICAL MATERIAL BY ATOMIC ABSORPTION

Dissolution

Method

F

G

L

M

N

$\mathrm{R}$
No. Samples

2

1

2

2

2

4
A1 $1 \%$ )

GXR-1 GXR-2 $\underline{\underline{\text { GXR-3 }}}$ GXR-4

$\begin{array}{llllll}3.7 & 16.3 & 6.19 & 7.46 & 19.2 & 15.6\end{array}$

$\begin{array}{llllll}3.62 & 18.7 & 5.92 & 7.04 & 21.7 & 16.4\end{array}$

$\begin{array}{llllll}3.9 & 17.8 & 7.14 & 7.09 & 21.2 & 16.5\end{array}$

$\begin{array}{llllll}0.52 & 5.19 & 2.64 & 4.74 & 5.45 & 7.62\end{array}$

$\begin{array}{llllll}3.22 & 18.8 & 6.61 & 6.40 & 22.1 & 18.4\end{array}$

$\begin{array}{llllll}1.18 & 7.47 & 3.32 & 5.77 & 7.25 & 9.68\end{array}$ 
TABLE VII: INFLUENCE OF DISSOLUTION METHOD CN THE DETERMINATION OF SILVER IN GEOLOGICAL MATRICES BY ATOMIC ABSORPTION

\begin{tabular}{|c|c|c|c|c|c|c|c|}
\hline \multirow{2}{*}{$\begin{array}{c}\text { Dissolution } \\
\text { Method }\end{array}$} & \multirow{2}{*}{$\begin{array}{c}\text { No. } \\
\text { Samples }\end{array}$} & \multicolumn{6}{|c|}{$\mathrm{Ag}(\mathrm{ppm})$} \\
\hline & & GXR-1 & $G \times R-2$ & GXR-3 & GXR-4 & GXR-5 & GXR-6 \\
\hline A & 28 & $28.5 \pm 11.5$ & $15 \pm 6$ & $2.8 \pm 0.3$ & $3.6 \pm 0.9$ & $2.0 \pm 1.7$ & $1.7 \pm 0.4$ \\
\hline B & 6 & $5.5 \pm 3.0$ & $8.9+4.8$ & $2.1 \pm 0.5$ & $3.7 \pm 0.4$ & $2.0 \pm 1.1$ & $1.6 \pm 0.5$ \\
\hline$E$ & 16 & $38 \pm 6$ & $20 \pm 2$ & $4 \pm 1$ & $6 \pm 1$ & $3 \pm 1$ & $3 \pm 1$ \\
\hline$H$ & 43 & $31 \pm 5$ & $19 \pm 8$ & $2.6 \pm 1.0$ & $4.2 \pm 0.8$ & $1.6 \pm 1.1$ & $1.4 \pm 0.6$ \\
\hline I & 38 & $26 \pm 12$ & $12 \pm 7$ & $2.9 \pm 1.0$ & $4.2 \pm 1.2$ & $1.6 \pm 0.5$ & $2.1 \pm 1.5$ \\
\hline$R$ & 19 & $31 \pm 6$ & $19 \pm 3$ & $2.5 \pm 1.0$ & $4.4 \pm 1.3$ & $1.8 \pm 0.5$ & $2 \pm 2$ \\
\hline
\end{tabular}


TABLE VIII: INFLUENCE OF DISSOLUTION METHOD ON THE DETERMINATION OF CALCIUM

IN GEOLOGICAL MATERIALS BY ATOMIC ABSORPTION

$\mathrm{Ca}(\%)$

\begin{tabular}{|c|c|c|c|c|c|c|c|}
\hline \multirow[b]{2}{*}{$\begin{array}{l}\text { Dissolution } \\
\text { Method }\end{array}$} & \multirow{2}{*}{$\begin{array}{c}\text { No. } \\
\text { Samples }\end{array}$} & & & & & & \\
\hline & & GXR-1 & GXR-2 & GXR-3 & GXR-4 & GXR-5 & GXR-6 \\
\hline G & 4 & $0.87 \pm 0.07$ & $0.93 \pm 0.07$ & $13.8 \pm 0.2$ & $1.00 \pm 0.08$ & $0.79 \pm 0.11$ & $0.23 \pm 0.04$ \\
\hline I & 14 & $0.56 \pm 0.14$ & $0.34 \pm 0.19$ & $13.8 \pm 1.6$ & $0.42 \pm 0.17$ & $0.29 \pm 0.18$ & $0.024 \rightarrow 0.14$ \\
\hline$L$ & 2 & 1.1 & 0.97 & 15.4 & 1.1 & 0.90 & 0.20 \\
\hline M & 8 & $0.93 \pm 0.17$ & $0.88 \pm 0.19$ & $12.1 \pm 0.7$ & $1.06 \pm 0.13$ & $0.85 \pm 0.14$ & $0.32 \pm 0.12$ \\
\hline N & 2 & 1.2 & 0.92 & 13.0 & 1.1 & 1.0 & 0.30 \\
\hline $\mathbf{R}$ & 8 & $0.93 \pm 0.05$ & $0.91 \pm 0.11$ & $6.9 \rightarrow 13.9$ & $1.06 \pm 0.12$ & $0.81 \pm 0.07$ & $0.21 \rightarrow 0.90$ \\
\hline
\end{tabular}


TABLE IX: INFLUENCE OF DISSOLUTION METHOD ON THE DETERMINATION OF IRON IN GEOLOGICAL MATERIALS BY ATOMIC ABSORPTION

\begin{tabular}{|c|c|c|c|c|c|c|c|}
\hline \multirow{2}{*}{$\begin{array}{l}\text { Dissolution } \\
\text { Method }\end{array}$} & \multirow{2}{*}{ Samples } & \multicolumn{6}{|c|}{$\mathrm{Fe}\left(\begin{array}{l}(\%) \\
0\end{array}\right)$} \\
\hline & & EXR-1 & GXR-2 & GXR-3 & $G \times R-4$ & GXR-5 & GXR-6 \\
\hline A & 24 & $17.0 \pm 1.5$ & $1.56 \pm 0.15$ & $13.3 \pm 3.9$ & $2.83 \pm 0.16$ & $3.02 \pm 0.21$ & $4.96 \pm 0.38$ \\
\hline$D$ & 2 & $>20$ & 2.10 & - & 3.00 & 3.81 & 6.30 \\
\hline $\mathrm{E}$ & 2 & 23.0 & 2.30 & 13.4 & 4.00 & 3.80 & 5.70 \\
\hline$F$ & 4 & $24.8 \pm 1.9$ & $2.08 \pm 0.10$ & $19.6 \pm 0.5$ & $2.94 \pm 0.18$ & $3.33 \pm 0.27$ & $5.37 \pm 0.43$ \\
\hline$G$ & 1 & 24.6 & 1.92 & 19.6 & 3.18 & 3.29 & 5.80 \\
\hline$H$ & $2-8$ & $23.8 \pm 1.7$ & 1.42 & $19.0 \pm 1.2$ & 2.88 & 2.94 & 5.37 \\
\hline I & 9 & $24.0 \pm 4.0$ & $1.84 \pm 0.13$ & $18.1 \pm 1.7$ & $2.90 \pm 0.85$ & $3.52 \pm 0.68$ & $5.43 \pm 0.45$ \\
\hline $\mathrm{N}$ & 6 & $25.9 \pm 0.7$ & $1.85 \pm 0.25$ & $20.0 \pm 0.1$ & $3.17 \pm 0.19$ & $3.66 \pm 0.08$ & $5.73 \pm 0.21$ \\
\hline$Q$ & 10 & $24.1 \pm 2.0$ & $1.78 \pm 0.13$ & $19.6 \pm 1.0$ & $3.13 \pm 0.15$ & $3.21 \pm 0.28$ & $5.60 \pm 0.33$ \\
\hline$R$ & $12-20$ & $25.2 \pm 1.5$ & $1.94 \pm 0.15$ & $19.2 \pm 1.3$ & $3.13 \pm 0.20$ & $3.51 \pm 0.29$ & $5.41 \pm 0.34$ \\
\hline$S$ & 12 & $24.9 \pm 1.7$ & $1.92 \pm 0.17$ & $19.4 \pm 1.8$ & $3.17 \pm 0.19$ & $3.51 \pm 0.22$ & $5.47 \pm 0.6$ \\
\hline
\end{tabular}


TABLE $X:$ INFLUENCE OF DISSOLUTION METHOD ON THE DETERMINATION OF MANGANESE

IN GEOLOGICAL MATERIALS BY ATOMIC ABSORPTION

\begin{tabular}{|c|c|c|c|c|c|c|c|}
\hline \multirow{2}{*}{$\begin{array}{l}\text { Dissolution } \\
\text { Method } \\
\end{array}$} & \multirow{2}{*}{$\begin{array}{c}\text { No. } \\
\text { Samples } \\
\end{array}$} & \multicolumn{6}{|c|}{$M n$ (ppm) } \\
\hline & & GXR-1 & GXR-2 & GXR-3 & $G \times R-4$ & GXR-5 & GXR-6 \\
\hline A & 7 & $810 \pm 100$ & $870 \pm 180$ & $3100 \pm 1000$ & $100 \pm 40$ & $160 \pm 60$ & $620 \pm 290$ \\
\hline C & 2 & 910 & 1000 & 22750 & 140 & 250 & 1050 \\
\hline D & 2 & 820 & 940 & 23000 & 160 & 280 & 940 \\
\hline$E$ & 6 & $810 \pm 160$ & $970 \pm 80$ & $9900 \pm 900$ & $160 \pm 10$ & $240 \pm 20$ & $1020 \pm 40$ \\
\hline$F$ & 2 & 900 & 980 & 23200 & 150 & 320 & 1040 \\
\hline G & 2 & 780 & 860 & 23400 & 210 & 300 & 1030 \\
\hline H & 22 & $820 \pm 140$ & $950 \pm 120$ & $20300 \pm 5900$ & $140 \pm 30$ & $240 \pm 40$ & $850 \pm 140$ \\
\hline I & 37 & $830 \pm 150$ & $910 \pm 140$ & $14200 \pm 8900$ & $130 \pm 20$ & $220 \pm 30$ & $920 \pm 180$ \\
\hline$L$ & 12 & $870 \pm 60$ & $950 \pm 70$ & $23100 \pm 1400$ & $170 \pm 20$ & $300 \pm 20$ & $910 \pm 160$ \\
\hline M & 2 & 880 & 850 & 18000 & 225 & 270 & 850 \\
\hline N & 8 & $940 \pm 50$ & $1000 \pm 60$ & $23800 \pm 500$ & $170 \pm 20$ & $310 \pm 30$ & $1100 \pm 80$ \\
\hline 0 & 1 & 700 & 800 & 17600 & 150 & 212 & 900 \\
\hline $\mathbf{P}$ & 2 & 1100 & 1200 & 32000 & 180 & 320 & 1200 \\
\hline Q & 10 & $970 \pm 140$ & $1000 \pm 220$ & $24900 \pm 4100$ & $140 \pm 30$ & $240 \pm 50$ & $1030 \pm 230$ \\
\hline$R$ & 20 & $900 \pm 20$ & $1000 \pm 70$ & $21700 \pm 3100$ & $150 \pm 10$ & $290 \pm 30$ & $1020 \pm 90$ \\
\hline $\mathrm{T}$ & 10 & $880 \pm 80$ & $950 \pm 100$ & $23000 \pm 1600$ & $150 \pm 10$ & $290 \pm 60$ & $910 \pm 160$ \\
\hline
\end{tabular}

